\title{
Spin gated GDR widths at moderate temperatures
}

\author{
Ish Mukul ${ }^{1, a}$, P. Sugathan ${ }^{1}$, J. Gehlot ${ }^{1}$, G. Mohanto ${ }^{1}$, A. K. Rhine Kumar ${ }^{2}$, I. Mazumdar ${ }^{3}$, Maninder Kaur ${ }^{4}$, N. \\ Madhavan $^{1}$, S. Nath ${ }^{1}$, R. Dubey ${ }^{1}$, T. Banerjee ${ }^{1}$, N. Saneesh ${ }^{1}$, D. A. Gothe ${ }^{3}$, P. Arumugam², and A. Roy ${ }^{1}$ \\ ${ }^{1}$ Inter University Accelerator Centre, Aruna Asaf Ali Marg, New Delhi, India \\ ${ }^{2}$ Department of Physics, Indian Institute of Technology Roorkee, Roorkee, Uttarakhand, India \\ ${ }^{3}$ Department of Nuclear and Atomic Physics, Tata Institute of Fundamental Research, Homi Bhabha Road, Mumbai, India \\ ${ }^{4}$ Department of Physics, Panjab University, Chandigarh, India
}

\begin{abstract}
.
We present the evolution of giant dipole resonance (GDR) width as a function of angular momentum in the compound nucleus ${ }^{144} \mathrm{Sm}$ in the temperature range of $1.5-2.0 \mathrm{MeV}$. The high energy $\gamma$ rays emitted from the decay of excited ${ }^{144} \mathrm{Sm}$ were measured using large $\mathrm{NaI}$ detector in coincidence with $4 \pi$ sum spin spectrometer. GDR widths were found to comply with thermal shape fluctuation model in this temperature range over a wide range of spin. Experimental widths tend to increase rapidly at high angular momentum values.
\end{abstract}

\section{Introduction}

Giant dipole resonance (GDR) built on excited states is an important tool in the study of nuclear structure as well as reaction dynamics. These resonances are observed with centroid energies in the range of $10-20 \mathrm{MeV}$ which corresponds to frequencies of the order of $10^{21} \mathrm{~Hz}$, thus making them the fastest known vibrations of a many body system. The structural properties of GDR could be related to different nuclear properties at high excitation energies, e.g. centroid energy is related to nuclear deformation and resonance width is related to the damping mechanism [1]. The behaviour of GDR widths with spin and temperature has been an interesting case and in past few decades several experiments have been performed in different mass regions to study the width evolution with spin [2]. For the sake of completeness of a study for a single nucleus, it is important to have sufficient number of data points over a large range of average spin values. A program to study the compound nucleus ${ }^{144} \mathrm{Sm}$ over a wide range of spin value at finite temperatures was undertaken at IUAC. In a previous experiment [3], we have measured the GDR widths as a function of spin in the temperature range of 1-1.5 MeV. In the present work, the behaviour of evolution of GDR widths over a wider range of angular momentum at two distinct temperatures is being reported. On the theoretical front, we have used two approaches to compare the experimental results. First approach is the thermal shape fluctuation model [4] which calculates potential energy surfaces, incorporating shell correction for this nucleus, and uses the same for calculation of GDR cross sections at finite temperature and spin. Second approach utilizes the phenomenological function proposed by Kusnezov et. al [5]

\footnotetext{
a e-mail: ishmukul@gmail.com
}

and calculates the GDR widths using an empirical relation. The first approach is found to be successful in reproducing the experimental GDR cross sections and the GDR widths for this nucleus at finite temperature and spin whereas second approach was used to check the consistency of this parametrization at different excitation energies for a single nucleus.

\section{Experimental details}

The experiment was performed using the 15UD Pelletron and superconducting linear accelerator facility at IUAC, New Delhi. ${ }^{28} \mathrm{Si}+{ }^{116} \mathrm{Cd}$ reaction was used to populate the compound nucleus ${ }^{144} \mathrm{Sm}$ at an excitation energy of $\sim 105 \mathrm{MeV}$. Pulsed beam, with a width of $83 \mathrm{ns,} \mathrm{was}$ utilized in this experiment. The target was rolled to $2.0 \mathrm{mg} \mathrm{cm}^{-2}$ using the rolling facility at target laboratory, IUAC. The target used in present experiment was self-supporting with an enrichment of more than $98.3 \%$. The $\gamma$ ray decay of excited ${ }^{144} \mathrm{Sm}$ was recorded using two spectrometers, namely, high energy gamma ray spectrometer (HIGRASP) [6], and $4 \pi$ sum-spin spectrometer [7]. The HIGRASP consists of $\mathrm{NaI}(\mathrm{Tl})$ detector in cylindrical geometry, with a dimension of $25.4 \mathrm{~cm} \times 30.5 \mathrm{~cm}$, surrounded by rectangular slabs of Plastic detectors. The plastic detectors served two purposes, namely, (a) to detect the $\gamma$ ray contribution from cosmic events, and (b) to detect the escape or scattered events from the NaI detector. The plastic signal was used as veto signal to suppress these events from actual GDR $\gamma$ ray contribution. The spin spectrometer consists of $32 \mathrm{NaI}(\mathrm{Tl})$ detectors in soccer ball geometry providing nearly $4 \pi$ solid angle coverage. In the present experiment, 29 detectors were used and each detector was kept at a threshold of $100 \mathrm{keV}$. The 

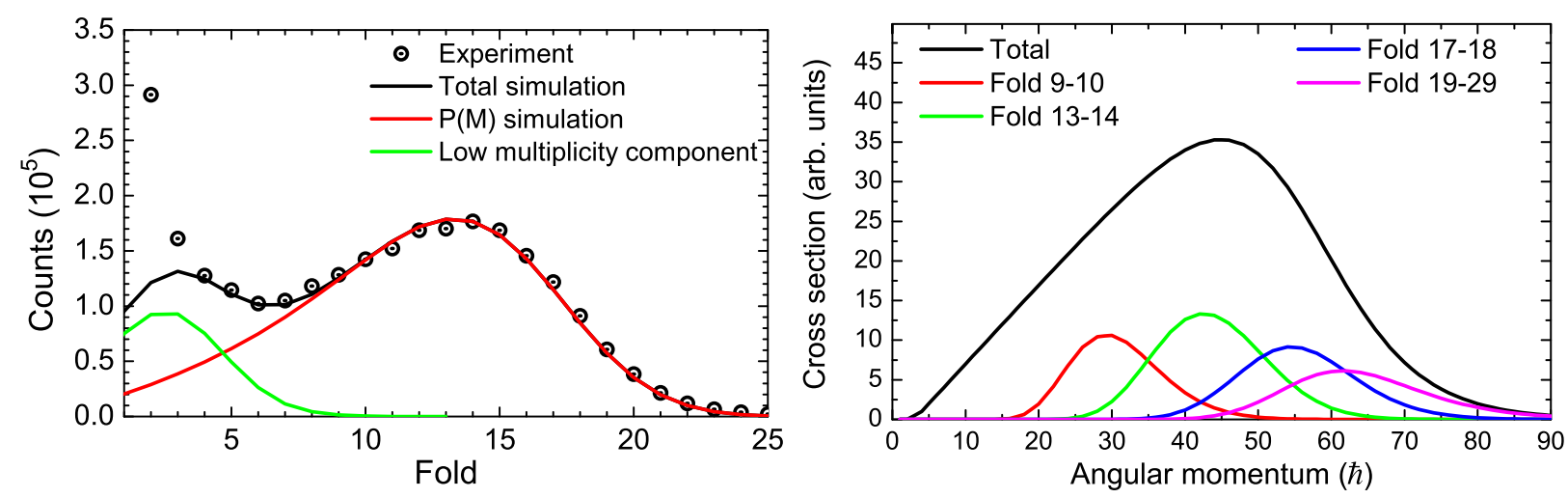

Figure 1. (Left) Experimental and simulated fold distribution in ${ }^{28} \mathrm{Si}+{ }^{116} \mathrm{Cd}$ reaction at $170 \mathrm{MeV}$ beam energy. (Right) Angular momentum distribution corresponding to different fold bins in the same reaction.

standard NIM based electronics was used for signal processing [8] in this experiment. High energy $\gamma$ rays were detected in HIGRASP, which was placed at $90^{\circ}$ with respect to beam line, in coincidence with low energy $\gamma$ rays detected in spin-spectrometer. Standard radioactive $\gamma$ ray sources were used for the calibration of detectors. The HIGRASP was calibrated for a full scale of $\sim 35 \mathrm{MeV}$ and its response function for different mono-energetic $\gamma$ rays was simulated by using GEANT4 simulation package [9]. The neutron- $\gamma$ separation was achieved by setting up a time of flight (TOF) between spin-spectrometer and HIGRASP. The pile-up effect in $\gamma$ ray spectra was reduced by standard zero cross-over technique. Data acquisition was performed using CAMAC based analog to digital (ADC). The software package used for on-line acquisition was candle [10] which was also used for off-line data reduction.

\section{Data reduction \& analysis}

The off-line data analysis for this experiment was a multistep process. First step involved the extraction of experimental GDR $\gamma$ ray energy spectra. This was accomplished by applying various gates on energy spectra, namely, $\gamma$ gate from $\mathrm{n}-\gamma$ TOF spectrum and pile-up gate from PU spectrum. The resulting $\gamma$ ray spectra was corrected for in-beam background and gated with different fold bins to get fold-gated $\gamma$ ray spectra. Second step involved the conversion of experimental fold distribution to angular momentum distributions. This conversion was performed by converting fold to multiplicity distribution and then relating multiplicity to angular momentum distribution. The response function of spin-spectrometer was generated using a recursive algorithm [11]. Inside an array of $N$ number of detectors, the probability $S(F, M)$ of $M$ number of $\gamma$ rays hitting $F$ number of detectors is given by

$S(F, M)=a S(F, M-1)+b S(F-1, M-1)+c S(F-2, M-1)$

where the variables $a, b$ and $c$ are given by

$$
a=1-(N-F) \omega(1+\epsilon F / N-1),
$$

$$
\begin{gathered}
b=(N-F+1) \omega(1-\epsilon(N-2 F+1) /(N-1)), \\
c=(N-F+2) \omega \epsilon((N-F+1) /(N-1)),
\end{gathered}
$$

and $\omega$ is the cross talk probability between two adjacent detectors and $\epsilon$ is the efficiency of individual detector. Simulated fold distributions were generated by folding this response function with an arbitrary multiplicity function using following equation,

$$
P(F)=\sum_{M=0}^{M_{\max }} S(F, M) P(M)
$$

We have used a modified Fermi function form for the multiplicity distribution given by

$$
P(M)=\frac{2 M+1}{1+\exp \left(\frac{M-M_{0}}{\delta M}\right)},
$$

where $M_{0}$ and $\delta M$ are free variables which were adjusted till the simulated fold distribution, obtained using Eq. 2, matches with the experimental fold distribution. The best values for $M_{0}$ and $\delta M$ were found to be at 25 and 3.1, respectively. A low multiplicity component, with $M_{0}=6.0$ and $\delta M=1.0$, was also added to fit the lower fold region. This contribution could be attributed to the nonfusion events occurring in the reaction. The multiplicity distributions corresponding to different fold bins were generated by $P(M)=S(F, M) P(F)$. The average of these multiplicity distributions could be related to average angular momentum as $\langle J\rangle=\langle M\rangle+C$, with $C=4$. The experimental and simulated fold distributions, and the angular momentum distributions corresponding to different fold bins are shown in Fig. 1.

The theoretical GDR $\gamma$ ray spectra were generated using code CASCADE [12] which is based on statistical model of decay of nucleus. This code was modified to include multiple Lorentzian GDR cross sections. The asymptotic level density parameter selected for present calculations was $\tilde{a}=\mathrm{A} / 8.5 \mathrm{MeV}^{-1}$. The theoretical spectra were compared with the experimental spectra, after folding with detector response generated using GEANT4, to extract GDR parameters. The code has an option of 

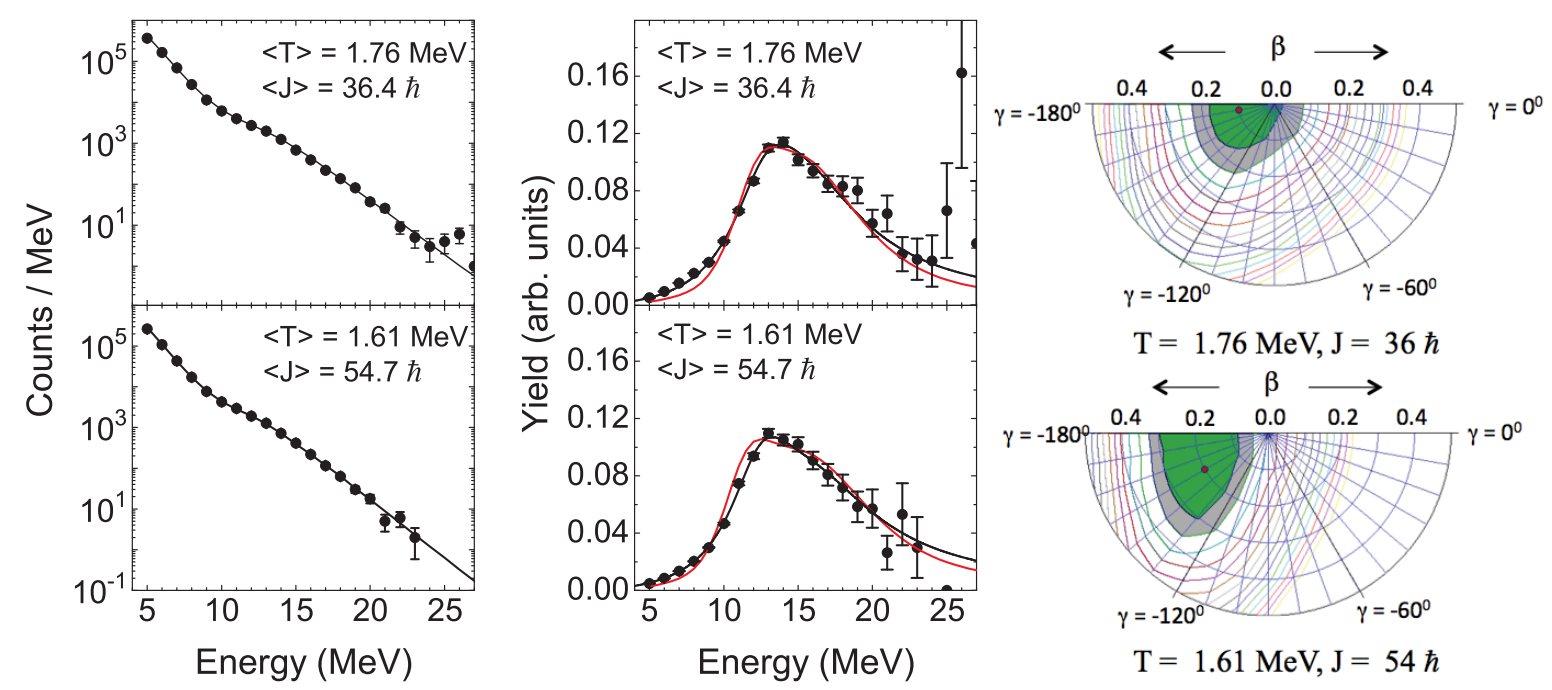

Figure 2. (Left) GDR $\gamma$ ray energy spectra at different average spin $(J)$ and temperature $(T)$ values, and (middle) the linearised plot for the same are shown. Red line represents GDR cross sections calculated by TSFM whereas black lines are CASCADE calculations. TSFM cross sections were normalized with respect to CASCADE cross sections. (Right) Potential energy surfaces of the nucleus ${ }^{144} \mathrm{Sm}$ at different $J$ and $T$ with a contour line spacing of $0.5 \mathrm{MeV}$. The filled circle represents the equilibrium shape and the first two minima are shaded.

changing multiple GDR parameters, namely, energy $\left(E_{i}\right)$, strength $\left(S_{i}\right)$ and width $\left(\Gamma_{i}\right)$, where $i$ is the $i$ th component of individual parameter. The goodness of fit was achieved by minimizing the $\chi^{2}$ in the energy range of $12-20 \mathrm{MeV}$. These parameters were used for generation of linearised plot as $Y_{\text {exp }} / Y_{t h} \times F_{L}\left(E_{\gamma}, E_{i}, \Gamma_{i}\right)$ where $Y_{\text {exp }}$ is the experimental yield, $Y_{\text {th }}$ is the CASCADE calculation folded with detector response and

$$
F_{L}\left(E_{\gamma}, E_{i}, \Gamma_{i}\right)=\sum_{i} \frac{S_{i} \Gamma_{i}^{2} E_{\gamma}^{2}}{\left(E_{\gamma}^{2}-E_{i}^{2}\right)+\Gamma_{i}^{2} E_{\gamma}^{2}} .
$$

The GDR $\gamma$ ray spectra at two different temperature and spin combination and their corresponding linearised plots are shown in Fig. 2.

\subsection{Theoretical calculations}

The nucleus populated at high excitation energy experiences thermal fluctuations which leads to fluctuation of nuclear surface due to orientation of rotational axis. The effective GDR cross sections, in this case, are sensitive to relative time scales for shape rearrangements. Thus, it is important to incorporate such fluctuations in TSFM while calculating the GDR cross sections [4]. The average value of GDR cross-section $(\sigma)$ is calculated as

$$
\langle\sigma\rangle_{\beta, \gamma}=\frac{\int \mathcal{D}[\alpha] \exp \{-F(T, J ; \beta, \gamma) / T\} \mathfrak{J}_{\mathrm{TOT}} \sigma(\beta, \gamma)}{\int \mathcal{D}[\alpha] \exp \{-F(T, J ; \beta, \gamma) / T\} \mathfrak{J}_{\mathrm{TOT}}}
$$

where $\mathcal{D}[\alpha]=\beta^{4}|\sin 3 \gamma| d \beta d \gamma$, and $\mathfrak{J}_{\text {TOT }}$ represents the moment of inertia inclusive of shell and surface diffuseness corrections. The free energy surfaces $(F)$ for this nucleus, at the extracted experimental temperature $(T)$ and spin $(J)$ values, were calculated using the Finite Temperature Cranked Nilsson-Strutinsky Method after applying the appropriate shell corrections. The GDR cross section were calculated for a fixed deformation in $\beta, \gamma$ space by considering an anisotropic harmonic oscillator potential with separable dipole-dipole interaction, where the GDR Hamiltonian could be written as

$$
H=H_{\mathrm{osc}}+\eta D^{\dagger} D,
$$

with the parameter $\eta$ represents the isovector component of the proton and neutron average field. The value of parameter $\eta$ was fixed at 3.0 for ${ }^{144} \mathrm{Sm}$ nucleus. The potential energy surfaces calculated using this formalism are shown in Fig 2. The GDR cross sections calculated for same $T$ and $J$ values, compared with experimental cross sections, are shown in same figure (represented by red solid line).

In another approach, Kusnezov et al. [5] have suggested that the GDR width for a nucleus of mass number $A$ at a finite $T$ and $J$ could be calculated using the empirical relation,

$$
\Gamma(T, J)=\Gamma(T, 0)\left(1+\frac{1.8}{1+\exp \left(\frac{1.3-\xi}{0.2}\right)}\right)^{\frac{4 T_{0}}{T+3 T_{0}}},
$$

where

$$
\Gamma(T, 0)=\left(6.45-\frac{A}{100}\right) \ln \left(1+\frac{T}{T_{0}}\right)+\Gamma_{0}(A) .
$$

In Eq. 7, $\Gamma_{0}(A)$ is the ground state width which is generally adjusted to match with the experimental width available at low spin value, and $T_{0}$ is the reference temperature chosen as $1 \mathrm{MeV}$. In these calculations, $\Gamma_{0}$ was fixed at $3.8 \mathrm{MeV}$, which is the same value used in the analysis for previous data [3]. 


\section{Results}

The experimental GDR widths $(\Gamma(T, J))$ were extracted by calculating the full width at half maxima (FWHM) of the linearised plots generated using CASCADE. The theoretical TSFM widths were calculated using FWHM of TSFM cross sections at different $T$ and $J$. The experimental, as well as, theoretical widths are presented as a function of $J$ at two different temperature bins of 1.5 and $1.8 \mathrm{MeV}$, and are shown in Fig. 3. At both $T$ values, the GDR widths show a rapid rise at high angular momentum values which signifies the strong coupling of spin degree of freedom with GDR at high angular momenta. The experimental widths are consistent with both models. In the case of Kusnezov parametrization, a few authors have reported a change in $\Gamma_{0}$ value at different excitation energies [13], whereas same value of $\Gamma_{0}$ is consistent for ${ }^{144} \mathrm{Sm}$ at different excitation energies.

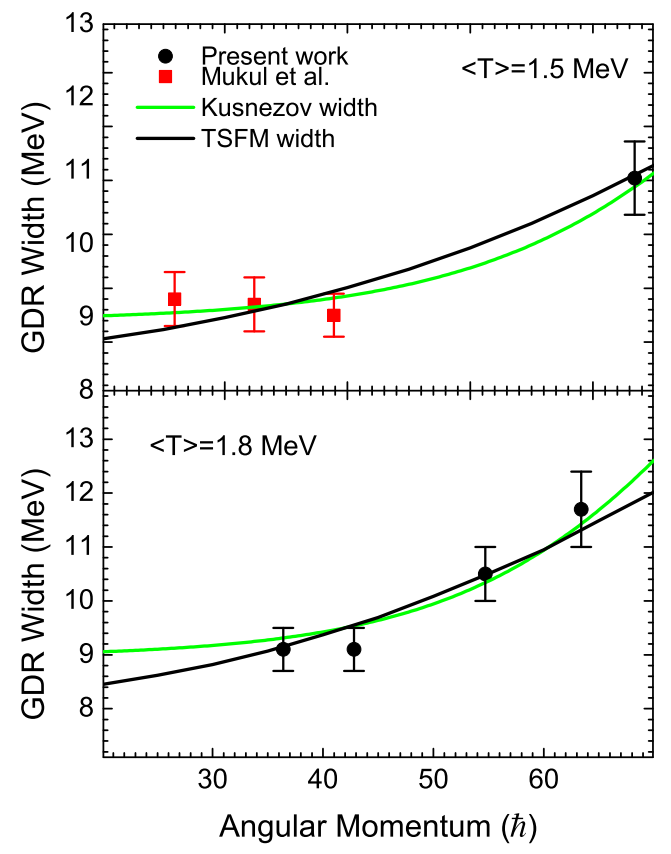

Figure 3. GDR width as a function of average angular momentum for different average temperature bins. Red squares are widths from previous measurement [3] and black circles represent present experimental data. Solid lines are theoretical calculations for different $T$ values using TSFM calculations (black line) and Kusnezov's parametrization (green line).

\section{Summary}

Spin gated GDR widths were extracted by exclusive measurement of GDR $\gamma$ rays from the decay of excited ${ }^{144} \mathrm{Sm}$ using ${ }^{28} \mathrm{Si}+{ }^{116} \mathrm{Cd}$ reaction at projectile energy of $170 \mathrm{MeV}$. The $\gamma$ rays were measured using large NaI detector in coincidence with low energy $\gamma$ ray multiplicity using $4 \pi$ spin spectrometer. The $\gamma$ ray decay spectrum was compared with statistical model of decay of compound nucleus and compared with the theoretical GDR cross sections under thermal shape fluctuation model. The extracted GDR widths shows uniform increase with temperature, consistent with the TSFM.

\section{Acknowledgements}

One of the author (IM) thanks University Grants Commission for providing financial support.

\section{References}

[1] J.J. Gaardhøje, Annu. Rev. Nucl. Part. Sci. 42, 483 (1992)

[2] S. Bhattacharya et al., Phys. Rev. C 78, 064601 (2008)

[3] I. Mukul et al., Phys. Rev. C 88, 024312 (2013)

[4] P. Arumugam et al., Phys. Rev. C 69, 054313 (2004)

[5] D. Kusnezov et al., Phys. Rev. Lett. 81, 542 (1998)

[6] I. Mazumdar et al., Nucl. Instrum. Methods Phys. Res. A 417, 297 (1998)

[7] G.A. Kumar et al., Nucl. Instrum. Methods Phys. Res. A 611, 76 (2009)

[8] I. Mukul et al., Eur. Phys. J. Web of Conference 63, 01020 (2013)

[9] S. Agostinelli et al., Nucl. Instrum. Methods Phys. Res. A 506, 250 (2003)

[10] B.P.A. Kumar et al., DAE Sympossium on Nuclear Physics, Kolkata 44B (2001)

[11] A. Maj et al., Nucl. Phys. A 571, 185 (1994)

[12] F. Pühlhofer, Nucl. Phys. A 280, 267 (1977)

[13] D.R. Chakrabarty et al., J. Phys. G: Nucl. Part. Phys. 37, 055105 (2010) 